\title{
ORIGINAL ARTICLE \\ Static standing, dynamic standing and spasticity in individuals with spinal cord injury
}

\author{
M Sadeghi ${ }^{1,2}$, J Mclvor ${ }^{3}$, H Finlayson $^{4}$ and B Sawatzky ${ }^{2,5}$
}

Study design: This was a cross-over efficacy study design.

Objective: To determine spasticity differences between static and dynamic standing training in individuals with spinal cord injury (SCl).

Setting: Vancouver, Canada.

Methods: Ten individuals with $\mathrm{SCl}$ who could stand with or without bracing or supports participated in both dynamic and static standing training (one session each, 2 days apart) using a Segway. The primary outcome was spasticity as measured by Visual Analog Scale (VAS), Modified Ashworth Scale (MAS) and electromyography (EMG) of the quadriceps, hamstrings, adductors and gastrocnemius.

Results: There was no statistically detectable difference in spasticity between dynamic and static standing training in individuals with $\mathrm{SCl}$ as measured by VAS, MAS or EMG, although there was a trend towards decreased spasticity after the dynamic training.

Conclusion: There is no significant difference in spasticity outcomes between static and dynamic standing training on a Segway for individuals with $\mathrm{SCl}$.

Sponsorship: This research was funded by Natural Sciences and Engineering Research Council of Canada and International Collaboration on Repair Discoveries.

Spinal Cord (2016) 54, 376-382; doi:10.1038/sc.2015.160; published online 22 September 2015

\section{INTRODUCTION}

Spasticity is a long-term complication of spinal cord injury (SCI) that is experienced by $65-78 \%$ of individuals with SCI. ${ }^{1}$ The most commonly used definition of spasticity is a velocity-dependent increase in muscle tone (tonic stretch reflex) with exaggerated tendon jerks (phasic component of stretch reflex), clonus and increased extrinsic reflexes. ${ }^{2-5}$

Spasticity in SCI is mostly managed by three different modalities; medications, physical therapy, and surgical intervention. Antispastic medication use may be limited by side effects, including weakness and sedation. ${ }^{6}$ Focal chemodenervation with botulinum toxins is often effective for localized spasticity and has few side effects and rare drug reactions. However, botulinum toxins are expensive and the effects are temporary, typically lasting from 2 to 6 months. ${ }^{6}$ Surgical techniques are invasive and are usually reserved for complications of spasticity such as contractures and as one of the last spasticity treatment options. ${ }^{6}$ Physical therapy techniques are an essential component of spasticity management and have a beneficial role before, during and after pharmacological and surgical strategies. ${ }^{6}$ Stretches, passive lengthening, weight-bearing techniques, muscle strengthening, electrical stimulation, vibration, supported standing, splints and orthoses are the most common physical therapy techniques that are used to manage spasticity. ${ }^{6}$
Supported standing is a weight-bearing physical therapy that has been shown to have potential benefits from the early to late stages of rehabilitation after SCI. ${ }^{7}$ Supported standing may improve posture, strengthen antigravity muscles, prepare muscles for gait training, retrain head and upper limb control, prevent contracture development and reduce spasticity. ${ }^{7,8}$ Upright standing and sensory inputs to the sole of the feet during standing may increase inhibition of the stretch reflex, reduce motor neuron excitability and subsequently reduce spasticity. ${ }^{7,9}$

Standing platforms are used in therapeutic and in-home settings owing to these proposed benefits. However, the static position can be boring, leading to lack of use. The Segway is a self-balancing, electricpowered personal transporter that an individual with SCI can stand on with or without long leg braces depending upon their level of injury. Individuals with different functional levels and varying degrees of strength, range of motion and balance found that they could easily and safely drive the Segway. ${ }^{10}$ In a small pilot study of eight individual with SCI, Boutilier et al. ${ }^{11}$ showed that spasticity decreased significantly immediately after using a Segway as measured by the Modified Ashworth Scale (MAS); however, no long-term benefits were observed over a month of use.

This raises the question as to whether the use of a Segway has additional benefits for managing spasticity over purely static standing.

${ }^{1}$ Department of Medicine, University of British Columbia, Vancouver, British Columbia, Canada; ${ }^{2}$ International Collaboration on Repair Discoveries, Vancouver, British Columbia, Canada; ${ }^{3}$ Department of Mechanical Engineering, University of British Columbia, Vancouver, British Columbia, Canada; ${ }^{4}$ Division of Physical Medicine and Rehabilitation, Department of Medicine, University of British Columbia, Vancouver, British Columbia, Canada and ${ }^{5}$ Department of Orthopaedics, University of British Columbia, Vancouver, British Columbia, Canada

Correspondence: Dr B Sawatzky, Department of Orthopaedics/ICORD, University of British Columbia, 818 West 10th Avenue, Vancouver, BC V5Z 1M9, Canada.

E-mail:bsawatzky@icord.org

Received 13 April 2015; revised 22 July 2015; accepted 10 August 2015; published online 22 September 2015 
The spasticity reduction mechanism might simply be standing on the Segway, but there may be additional mechanisms responsible for the reduction in spasticity with this type of training. The purpose of this study was to examine the effects of two different standing training interventions (dynamic and static) on spasticity to explore which type of training would reduce spasticity more. This study also aimed to demonstrate a novel method for analyzing electromyography (EMG) signals for this application in this population. This study hypothesized that dynamic standing training on the Segway would result in greater spasticity reduction when compared with static standing training as measured by MAS, Visual Analog Scale (VAS) and EMG.

\section{MATERIALS AND METHODS}

The study was carried out at the International Collaboration On Repair Discoveries (Vancouver, BC, Canada). Ethical approval was obtained from the University of British Columbia's Ethical Review Board before the beginning of the study.

\section{Inclusion criteria}

A total of 10 individuals living with SCI were recruited to participate in this study. To be considered for participation in the study, participants had to meet the following inclusion criteria; (1) age from 18 to 65 years, (2) SCI for $>1$ year, (3) spasticity of lower extremities (MAS $\geqslant 1$ ) for at least 1 month before participating in the study, (4) the ability to rise from sitting to standing with no more than moderate assistance from one person and/or with the use of long leg braces, (5) no autonomic dysreflexia based on their medical history, and (6) no acute urinary tract infection nor pressure ulcers.

\section{Study design}

This was a cross-over efficacy study design. The carry over effect from the previous standing training mostly lasted for a short period of time at the most to the next day. ${ }^{6,12}$ A 1-week washout period was chosen to attempt to eliminate any carry over effect from the previous training session.

\section{Outcome measures/study protocol}

Three outcome measures were used to assess the effects of the standing training on spasticity in the four muscle groups: the quadriceps, hamstrings, adductors, and gastrocnemius muscles. The primary outcome measure was the MAS (all muscles except adductors). ${ }^{13}$ The VAS ${ }^{13}$ and EMG were secondary outcome measures. EMG was measured, in part, to determine the best way to define muscle spasticity electrophysiologically.

The MAS is a subjective clinical assessment of muscle tone which is scored on a six-point scale $(0,1,+1,2,3,4)$ based on the resistance the examiner experiences through the passive range of motion. ${ }^{13,14}$ The VAS is a simple $10-\mathrm{cm}$ line self-report questionnaire on which the participants report their level of spasticity in each muscle group ranging from ' $0=$ no spasticity' to ' $10=$ the most imaginable spasticity. ${ }^{13}$ EMG is a repeatable outcome measure that records the reflexive electrical activity of the testing muscle against stretch to evaluate the muscle spasticity. ${ }^{14}$

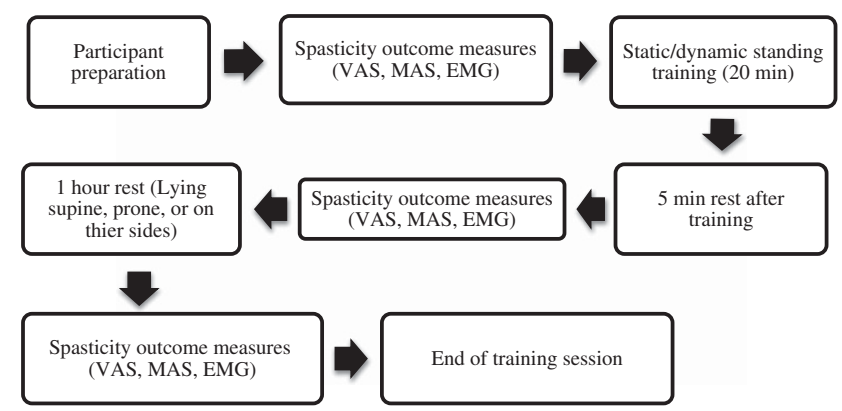

Figure 1 Protocol schematic.
Each participant came to the research laboratory three times. There was one familiarization session followed by two training sessions. As factors such as medication, bladder fullness, time of day and level of physical activity can affect spasticity, the participants were asked to come in at the same time for both training sessions and to maintain similar exercise regimes during the study period. All participants were asked to empty their bladder before each training session.

The participants' level of spasticity was tested during the familiarization session and they had 10 min of training on a Segway, before the actual dynamic training session. The participants were asked which leg had more spasticity and spasms. On the training day, four sites on the most affected leg were shaved to correspond with the quadriceps, hamstrings, adductors and medial gastrocnemius muscles. EMG electrodes were attached to the sites, with the ground electrode located on the medial malleolus of opposite leg. To measure the range of motion and joint velocity during MAS manoeuvres, knee and ankle electrogoniometers were also affixed to the knee and ankle.

The Segway has a small platform with two parallel wheels and a closed loop dynamic stability control system. The closed loop control system is controlled by gyroscopes and other sensors. Gyroscopes detect forward and backward movements of the rider that results in forward and backward movement of the Segway, respectively. The angle at which a person leans forward and backward controls the velocity of the Segway. Leaning forward results in a greater velocity and leaning backward results in a lower velocity. ${ }^{15}$

Both standing training sessions lasted for $20 \mathrm{~min}$ based on the standing training durations used in previous studies. ${ }^{11}$ The protocol schematic is shown in Figure 1. To ensure the safety of the participants during the training session, a harness system attached to a long ceiling track was used in the laboratory (Figure 2). For static standing, the Segway was securely fixed with four blocks underneath the platform. The dynamic standing was divided into $4 \times 5 \mathrm{~min}$ training: $0-5 \mathrm{~min}$ (slow forward and backward movements and stops), 5-10 min (fast forward and backward movements and stops), 10-15 min (narrow and wide turns to right and left), and 15-20 min (free to combine the training).

\section{Data analysis}

A custom interactive program was written in MATLAB (MathWorks Inc., Natick, MA, USA) to process the goniometers and the EMG data offline.

Data from the goniometers were converted from voltage to degrees using the equations calculated by the regression model. The equations are listed

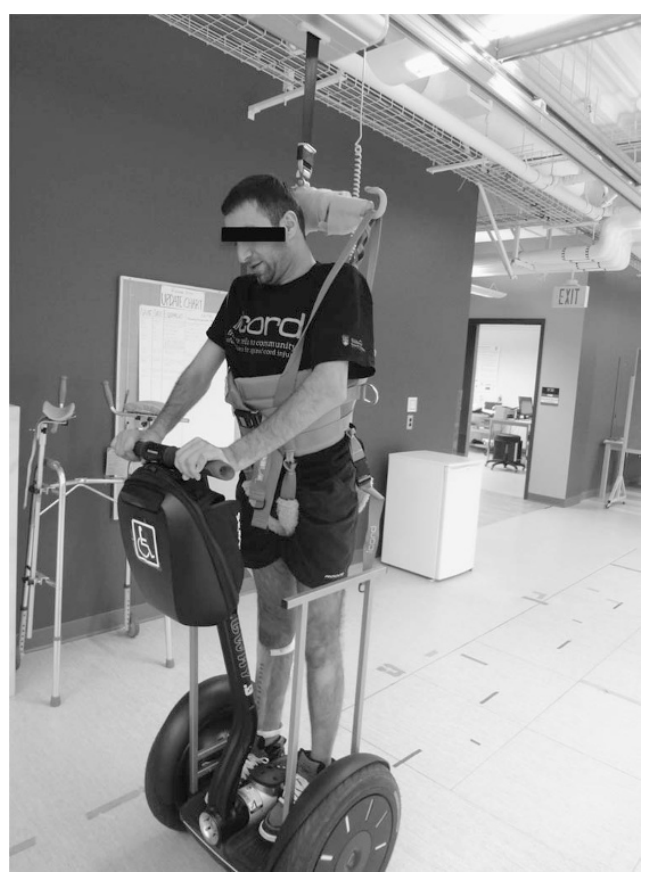

Figure 2 Dynamic standing training. 
below:

Anklegoniometer $_{(\text {degree }}=93 \times$ Averagevoltage $_{(\mathrm{V})}+(-233)$
Kneegoniometer $_{(}=93 \times$ Averagevoltage $_{(\mathrm{V})}+(-234)$

The goniometer data were filtered using a low pass filter with a cutoff frequency of $6 \mathrm{~Hz}$. Ankle and knee joint range of motion data were differentiated to calculate the angular speed $\left(\mathrm{deg} \mathrm{s}^{-1}\right)$ of each movement. An angular speed of $5 \mathrm{deg} \mathrm{s}^{-1}$ was chosen as the threshold to define the start and stop of the ankle and knee passive movements. Figure 3 shows a typical example of the knee angle and angular speed with the threshold used to define the onset and offset of movement.

EMG data were collected using a 16-channel Bagnoli system with a sampling frequency of $1000 \mathrm{~Hz}$ and stored digitally for processing offline. Before digitizing, EMG data were passed through a bandpass filter with a bandpass of $20-450 \mathrm{~Hz} \pm 10 \%$. Offline, the EMG data had several processing steps applied: removal of bias, rectification, application of a $60-\mathrm{Hz}$ notch filter to remove electrical noise, and application of a low pass filter with a cutoff frequency of $10 \mathrm{~Hz}$.

Processed EMG is typically normalized by scaling with a maximum voluntary isometric contraction (MVIC). However, this is problematic in individuals with a SCI, as there is a high probability of a weak MVIC and the recorded MVIC is not representative of the involuntary muscle activity in response to the stretch reflex. ${ }^{8}$ To compare between participants and between training sessions within a participant, an alternative method was required.

In lieu of MVIC, EMG data were normalized using integrated EMG (IEMG) and a standard length of time for each movement. The IEMG is defined as the area under the curve of the EMG signal. An ankle EMG processing duration of $1 \mathrm{~s}$ was chosen based on the average duration $(0.92 \pm 0.49 \mathrm{~s})$ of ankle dorsiflexion movement from the start to the stop point (Figure 4a). A knee EMG processing duration of $2 \mathrm{~s}$ was chosen based on the average duration of passive knee flexion $(2.13 \pm 0.82 \mathrm{~s})$ and knee extension $(1.94 \pm 0.60 \mathrm{~s})$ from the start to the stop point (Figure $4 \mathrm{~b}$ ). The IEMG of the baseline EMG (same duration for each movement) was calculated for each movement. The baseline IEMG was subtracted from the IEMG during the movement and that value was used for further analysis.

The IEMG changes $(\Delta)$ were calculated as the difference of the IEMG before and immediately after the standing training. This formula was also used to calculate the changes from before to $1 \mathrm{~h}$ after the standing training.

$$
\begin{aligned}
& \triangle \operatorname{IEMG}(\mathrm{B}-\mathrm{A})=\mathrm{IEMG}_{\text {before }}-\mathrm{IEMG}_{\text {after }} \\
& \triangle \mathrm{IEMG}(\mathrm{B}-\mathrm{H})=\mathrm{IEMG}_{\text {before }}-\mathrm{IEMG}_{\text {lhlater }}
\end{aligned}
$$

To compare the effects of static and dynamic standing condition on spasticity and muscle activity changes, this study used $\triangle \mathrm{IEMG}(\mathrm{B}-\mathrm{A})$ and $\triangle \mathrm{IEMG}(\mathrm{B}-\mathrm{H})$.

\section{Statistical analysis}

A $2 \times 3$ (condition $\times$ time) repeated-measures analysis of variance was used to compare VAS values to determine the difference between dynamic and static standing training effects on spasticity. The related-samples Friedman two-way analysis of variance by ranks statistical analysis method was used to compare the MAS scores between conditions. A $2 \times 2$ repeated-measures analysis of variance was used to assess changes in IEMG.

\section{Statement of ethics}

We certify that all applicable institutional and governmental regulations concerning the ethical use of human volunteers were followed during the course of this research.

\section{RESULTS}

\section{Population}

Ten individuals with SCI (nine males and one female) with a level of injury between $\mathrm{C} 3$ and $\mathrm{T} 6$ volunteered to participate in the study. The mean \pm s.d. age of the participants was $40.4 \pm 11.15$ years. The specific characteristics of these individuals are summarized in Table 1.

\section{Modified Ashworth Scale}

The MAS scores of all the participants are reported in Table 2. Table 3 presents the percentage of participants scoring for each MAS scores. There was no significant interaction effect between the static and dynamic standing training MAS scores over time (before, immediately after and $1 \mathrm{~h}$ later) for ankle dorsiflexion $(P=0.57)$, knee flexion $(P=0.24)$ and knee extension $(P=0.60)$. However, the average MAS scores of all passive movements showed a decreasing trend over time from before to immediately after and from before to $1 \mathrm{~h}$ later for both
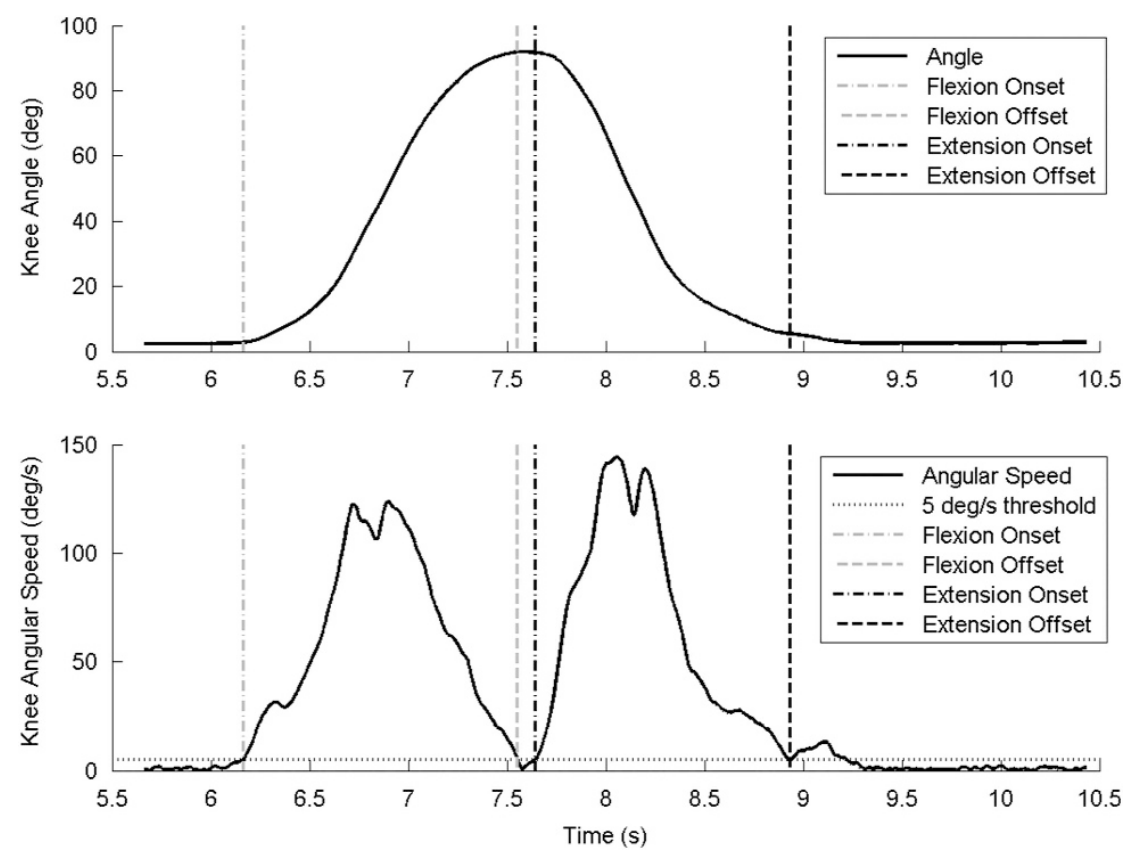

Figure 3 Knee goniometer processing. Top figure shows the knee angle (deg) and lower figure shows knee angular speed (deg s ${ }^{-1}$ ) during flexion/extension. 

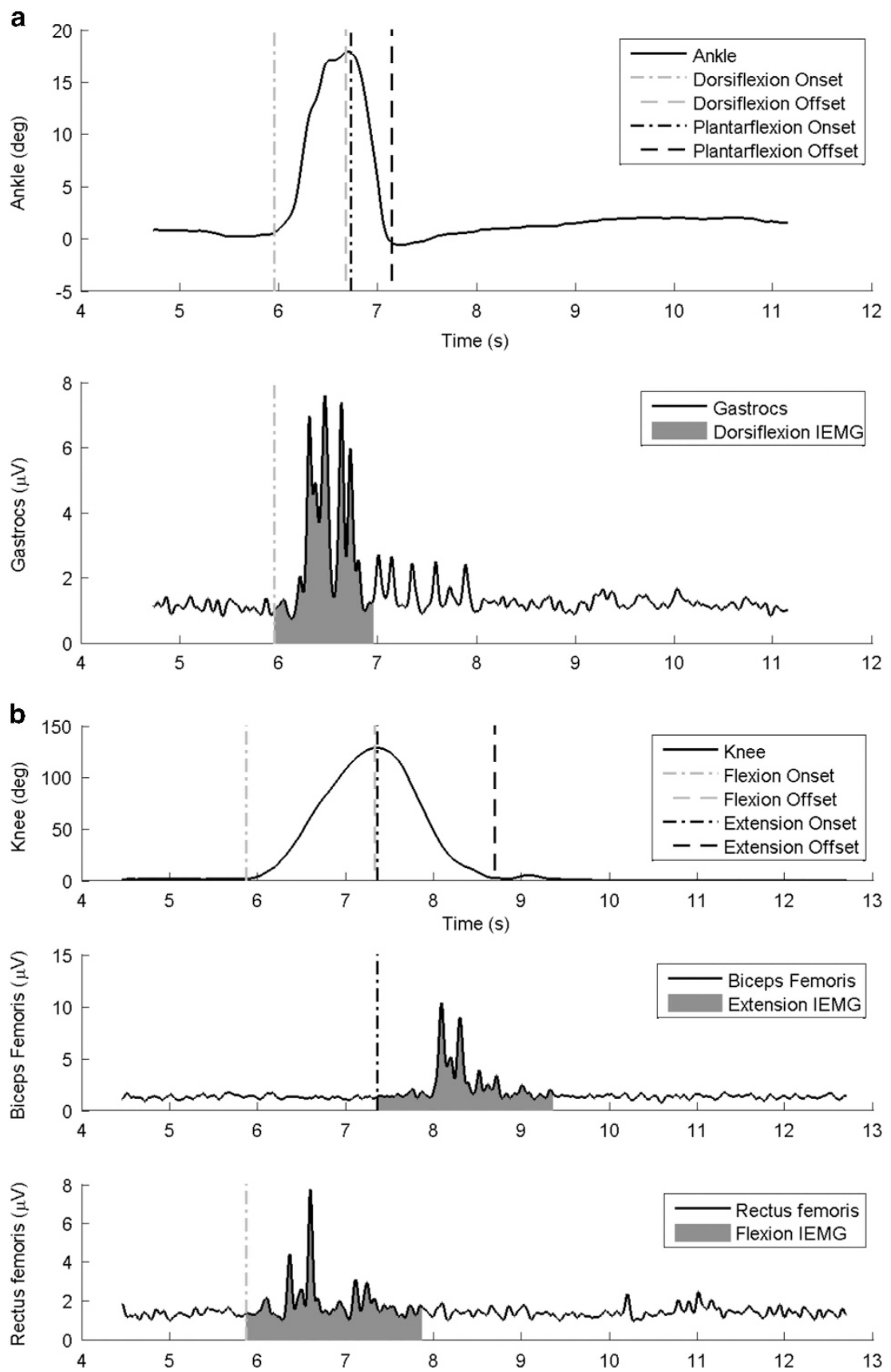

Figure 4 Integrated EMG. Ankle dorsiflexion integrated EMG (a) and knee flexion and extension integrated EMG (b) based on the goniometer start point of the passive movements.

the dynamic and static standing training but was not statistically significant.

\section{Visual Analog Scale}

The mean and s.d. of each tested muscle group is reported in Figure 5. There were decreasing trends from before to immediately after and from before to $1 \mathrm{~h}$ after both dynamic and static training; however, these changes did not reach statistical significance. There was no significant interaction effect between the static and dynamic standing training over time (before, immediately after and $1 \mathrm{~h}$ later; however, again trends were seen for the quadriceps $(P=0.10)$, hamstrings $(P=0.07)$ and calf $(P=0.15)$ muscle groups (Figure 5$)$.

\section{Electromyography}

The mean and s.d. of each tested muscle group regarding the passive movement that causes stretch in that muscle is reported in Table 4. There was no significant interaction effect $(P=0.28)$, standing main effect $(P=0.42)$ and main effect of time $(P=0.30)$ for the gastrocnemius muscle over ankle dorsiflextion. There was no significant interaction effect $(P=0.26)$, standing main effect $(P=0.25)$ and main effect of time $(P=0.16)$ for the quadriceps muscle over knee flexion. There was no significant interaction effect $(P=0.84)$, standing main effect $(P=0.22)$ and main effect of time $(P=0.30)$ for the hamstrings muscle over knee extension. There was no significant difference between dynamic and static standing training on tested muscles that were not 
Table 1 Spinal cord injury participants' characteristics

\begin{tabular}{|c|c|c|c|c|c|c|c|c|}
\hline No. & Age (years) & Gender & Year of injury & Injury level & AIS & Complete/incomplete injury & Spasticity meds & Mobility \\
\hline 1 & 42 & M & 2009 & C4-C5 & $\mathrm{D}$ & Incomplete & No & Walk \\
\hline 2 & 42 & M & 1987 & C4-C5 & $\mathrm{D}$ & Incomplete & No & Power WC \\
\hline 3 & 30 & M & 2003 & $\mathrm{~T} 4$ & A & Complete & No & Manual WC \\
\hline 4 & 31 & $M$ & 2002 & T5-T6 & C & Incomplete & No & Power WC \\
\hline 5 & 31 & M & 2003 & C5-C6 & $\mathrm{D}$ & Incomplete & No & Power WC \\
\hline 6 & 47 & $M$ & 2010 & C3 & $\mathrm{D}$ & Incomplete & Baclofen/Gabapentine & Walk \\
\hline 7 & 55 & M & 2011 & C5-C7 & $\mathrm{D}$ & Incomplete & Baclofen & Manual WC \\
\hline 8 & 36 & $M$ & 1993 & C7 & $\mathrm{C}$ & Incomplete & No & Manual WC \\
\hline 9 & 61 & $M$ & 2012 & $\mathrm{C} 4-\mathrm{C} 5$ & $\mathrm{D}$ & Incomplete & Nabilone & Walk \\
\hline 10 & 29 & $\mathrm{~F}$ & 2008 & C4-C5 & $\mathrm{D}$ & Incomplete & No & Walk \\
\hline
\end{tabular}

Abbreviations: AIS, Abbreviated Injury Scale; F, female; M, male; WC, wheelchair.

Table 2 Modified Ashworth Scale outcome measure

\begin{tabular}{|c|c|c|c|c|c|c|c|c|c|c|c|c|c|c|c|c|c|c|}
\hline \multirow[t]{3}{*}{ No. } & \multicolumn{9}{|c|}{ Dynamic standing } & \multicolumn{9}{|c|}{ Static Standing } \\
\hline & \multicolumn{3}{|c|}{ Dorsiflexion } & \multicolumn{3}{|c|}{ Knee flexion } & \multicolumn{3}{|c|}{ Knee extension } & \multicolumn{3}{|c|}{ Dorsiflexion } & \multicolumn{3}{|c|}{ Knee flexion } & \multicolumn{3}{|c|}{ Knee extension } \\
\hline & $B$ & $A$ & $1 H$ & $B$ & $A$ & $1 H$ & $B$ & $A$ & $1 H$ & $B$ & $A$ & $1 H$ & $B$ & $A$ & $1 H$ & $B$ & $A$ & $1 H$ \\
\hline 1 & 1 & 0 & 0 & $1+$ & $1+$ & $1+$ & $1+$ & $1+$ & $1+$ & 1 & 1 & 1 & $1+$ & $1+$ & $1+$ & $1+$ & $1+$ & $1+$ \\
\hline 2 & 1 & $1+$ & 1 & $1+$ & 2 & 2 & 1 & 1 & $1+$ & 1 & $1+$ & 1 & $1+$ & 2 & 2 & 1 & $1+$ & 1 \\
\hline 3 & 1 & 0 & 1 & 1 & 0 & 0 & 1 & 0 & 0 & 1 & 1 & 1 & $1+$ & 1 & 1 & 1 & 0 & 0 \\
\hline 4 & 2 & $1+$ & $1+$ & 2 & 0 & $1+$ & $1+$ & 0 & 1 & $1+$ & 1 & 1 & 2 & $1+$ & $1+$ & 1 & 0 & 0 \\
\hline 5 & 2 & $1+$ & 2 & $1+$ & 1 & 1 & $1+$ & 1 & 1 & 2 & 2 & 3 & 1 & 1 & 1 & 1 & 1 & 1 \\
\hline 6 & $1+$ & 1 & 1 & 2 & $1+$ & $1+$ & $1+$ & $1+$ & $1+$ & 1 & 1 & $1+$ & 1 & 1 & 1 & 1 & 1 & $1+$ \\
\hline 7 & $1+$ & 2 & 1 & $1+$ & 1 & 1 & $1+$ & $1+$ & $1+$ & $1+$ & 2 & $1+$ & $1+$ & $1+$ & 1 & $1+$ & $1+$ & 1 \\
\hline 8 & 2 & 3 & 2 & 2 & 2 & 2 & 2 & $1+$ & 1 & 3 & 2 & 2 & 2 & $1+$ & $1+$ & $1+$ & 1 & 2 \\
\hline 9 & 2 & 2 & 2 & 1 & 0 & $1+$ & $1+$ & 0 & 0 & 2 & 2 & $1+$ & $1+$ & 0 & 0 & $1+$ & 0 & 0 \\
\hline 10 & 2 & $1+$ & 2 & 2 & 2 & 2 & 1 & 1 & 1 & 2 & $1+$ & $1+$ & 2 & 2 & 2 & 1 & 1 & 1 \\
\hline
\end{tabular}

Abbreviations: $\mathrm{A}$, after; $\mathrm{B}$, before; $1 \mathrm{H} ; 1$ hour.

Table 3 Percentage of Modified Ashworth Scale outcome measure

\begin{tabular}{|c|c|c|c|c|c|c|c|c|c|c|c|c|c|c|c|c|c|c|}
\hline \multirow[t]{3}{*}{ Score } & \multicolumn{9}{|c|}{ Dynamic standing } & \multicolumn{9}{|c|}{ Static standing } \\
\hline & \multicolumn{3}{|c|}{ Dorsiflexion } & \multicolumn{3}{|c|}{ Knee flexion } & \multicolumn{3}{|c|}{ Knee extension } & \multicolumn{3}{|c|}{ Dorsiflexion } & \multicolumn{3}{|c|}{ Knee flexion } & \multicolumn{3}{|c|}{ Knee extension } \\
\hline & $B$ & $A$ & $1 H$ & $B$ & $A$ & $1 H$ & $B$ & $A$ & $1 H$ & $B$ & $A$ & $1 H$ & $B$ & $A$ & $1 H$ & $B$ & $A$ & $1 H$ \\
\hline 0 & 0 & 20 & 10 & 0 & 30 & 10 & 0 & 30 & 20 & 0 & 0 & 0 & 0 & 10 & 10 & 0 & 30 & 30 \\
\hline 1 & 30 & 10 & 40 & 20 & 20 & 20 & 30 & 30 & 40 & 40 & 40 & 40 & 20 & 30 & 40 & 60 & 40 & 40 \\
\hline $1+$ & 20 & 40 & 10 & 40 & 20 & 40 & 60 & 40 & 40 & 20 & 20 & 40 & 50 & 40 & 30 & 40 & 30 & 20 \\
\hline 2 & 50 & 20 & 40 & 40 & 30 & 30 & 10 & 0 & 0 & 30 & 40 & 10 & 30 & 20 & 20 & 0 & 0 & 10 \\
\hline 3 & 0 & 10 & 0 & 0 & 0 & 0 & 0 & 0 & 0 & 10 & 0 & 10 & 0 & 0 & 0 & 0 & 0 & 0 \\
\hline
\end{tabular}

Abbreviations: $\mathrm{A}$, after $\mathrm{B}$, before; $1 \mathrm{H}, 1$ hour.

Numbers are in percentage (\%).

primarily stretched (for example, adductors during ankle dorsiflexion or knee flexion/extension) during each passive movement. The data from these non-primary muscles are not reported here. The graphical representation of the EMG suggested a possible decreasing trend from before to immediately after and before to $1 \mathrm{~h}$ later of dynamic standing training versus static standing training for muscles (Figure 6).

\section{DISCUSSION}

The main purpose of this study was to assess to what degree two different standing conditions, dynamic (on the Segway) and static standing, may decrease spasticity in individuals with SCI. The results did not support the presence of a statistically significant additional spasticity reduction using the dynamic standing on the Segway; however, there was a trend toward decreased spasticity after both conditions. This latter finding supports previous work that has shown that both static and dynamic standing training are helpful for spasticity management in individuals with SCI. ${ }^{6,11,16,17}$

Although there was a decreasing trend for both static and dynamic standing training, there was no statistically significant difference between these two interventions as measured by VAS. There was a 
non-statistically significant change of SCI-SET (Spasticity Evaluation Tool) scores over a 1 -month $(3 \times$ per week) dynamic training program with the Segway from the beginning to the last training session in individuals with SCI $(n=8) .{ }^{11}$ SCI-SET is a global measure of spasticity that looks at both positive and negative effects of spasticity on function. As this present study was focusing on specific muscle groups, the participants judged four different lower extremity muscle groups separately. Although it was hard for the study participants to imagine their muscles' spasticity level individually, it was more appropriate for assessing how each muscle group spasticity level responded to the standing interventions. Although not statistically significant, both our current study and the previously published study $^{11}$ did show trends in reducing perceived spasticity using a Segway despite their small sample size.

Similar to VAS score, although MAS scores were reduced over time after both standing interventions, there was no statistically significant difference between the static and dynamic standing training MAS scores. Both static and dynamic standing training have been shown to reduce MAS measures of spasticity in individuals with different upper motor neuron disorders. ${ }^{6,11,17}$ However, no statistically significant difference was found between the two training conditions. Again this highlights the point that both methods may be effective in contributing to spasticity management in SCI but that one method is not superior for reducing spasticity.

The muscles measured using a passive movement technique did not show statistically significant change of EMG activity between the training methods over time; however, the gastrocnemius and hamstrings muscle activity appears to decrease more after the dynamic versus static standing training for ankle dorsiflexion and knee extension. There was a similar trend in other muscles.

The quadriceps muscle activity was measured by a single surface electrode located over the rectus femoris muscle. Although the authors did their best to locate the EMG electrodes, crosstalk between the vastus medialis and vastus lateralis likely made it difficult to isolate activity from rectus femoris. Thus all results are reported as quadriceps rather than specifying rectus femoris.

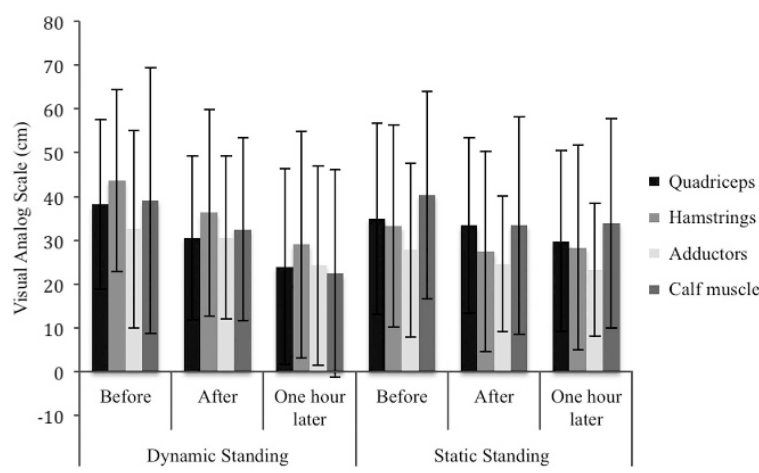

Figure 5 Visual Analog Scale outcome measure results.
This study also attempted to address the inherent challenges in analyzing and comparing the EMG data of individuals with SCI by using an IEMG normalization method. The challenge of using EMG to define spasticity is not only limited to lack of providing MVIC and a representative of the involuntary muscle activity in response to the stretch reflex. ${ }^{8}$ There is currently no operational definition for spasticity based on EMG. However, some researchers have defined spasms based on EMG measurements. For instance, spastic response has been defined as (1) any non-zero EMG activity resulting from passive maneuvers and/or (2) any EMG activity amplitude of $\geqslant 4$ times the baseline amplitude with a duration of $>5 \mathrm{~s} .{ }^{18,19}$ Although these previous definitions may be satisfactory to classify spasms and the presence of spasticity in individuals with upper motor neuron disorders, it has not been validated as an outcome measure to compare muscle tone changes after two different interventions. Because of the limitations in defining spasticity by EMG, this outcome measure was used only to determine involuntary muscle activity changes over time with standing training. The proposed EMG normalization method used in this study was able to assess relative differences in muscle activity after standing interventions.

Using IEMG to assess relative changes in spasticity has links with other work using IEMG and H-reflex techniques. Several studies have
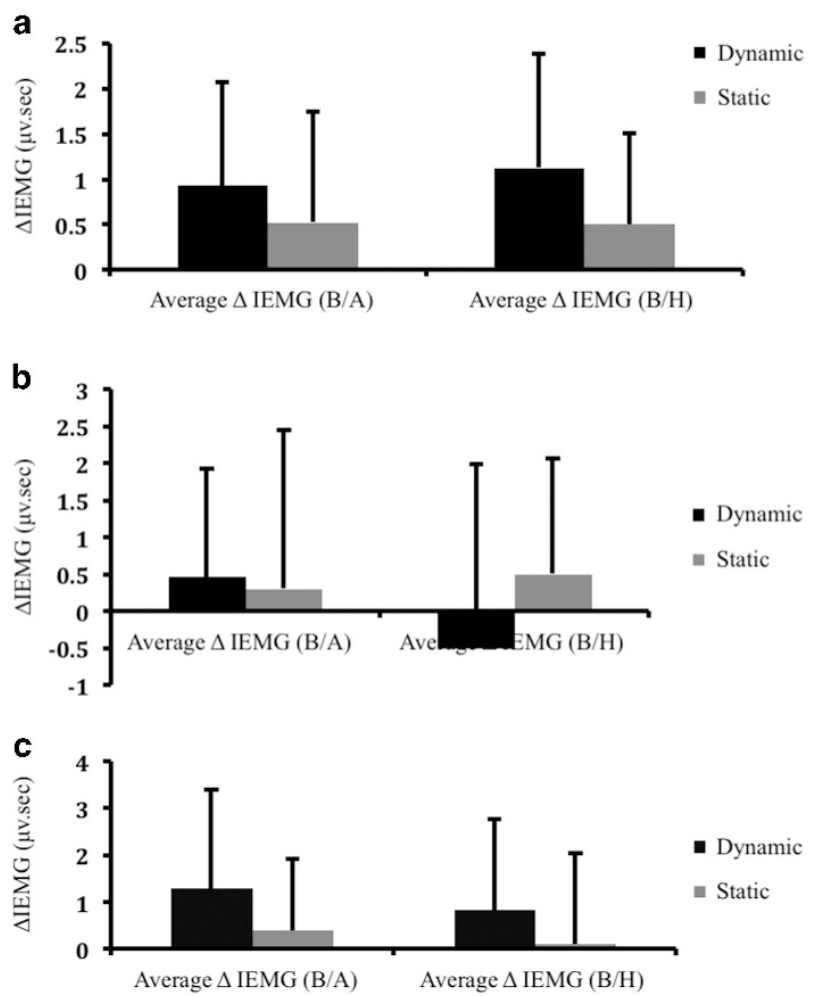

Figure 6 IIEMG changes. Gastrocnemius (a), quadriceps (b), hamstrings (c).

Table $4 \mathrm{SCl}$ group average $\triangle \mathrm{IEMG}$ changes for the primary muscles

\begin{tabular}{lccc}
\hline Muscle & Average $\Delta / E M G(B-A) /$ dynamic & Average $\Delta / E M G(B-A) /$ static & Average $\Delta / E M G(B-H) /$ dynamic \\
& $(\mu v . s e c)$ & $(\mu v . s e c)$ & $(\mu v . s e c)$ \\
\hline Gastrocnemius & $0.93 \pm 1.14$ & $0.52 \pm 1.22$ & $1.12 \pm 1.26$ \\
Quadriceps & $0.46 \pm 1.46$ & $0.30 \pm 2.14$ & $-0.50 \pm 2.48$ \\
Hamstrings & $1.28 \pm 2.12$ & $0.40 \pm 1.52$ & $0.50 \pm 1.00$ \\
\hline
\end{tabular}

Abbreviations: (B-A), before-after; (B-H), before-1 h later; IEMG, integrated electromyography; SCI, spinal cord injury. 
found that standing in a frame setting produced significantly lower $\mathrm{H} / \mathrm{M}$ ratio and maximum H-reflex amplitude versus the sitting position; the authors suggested that the stretch reflex excitability reduction and consequently spasticity reduction after standing training in individuals with SCI. ${ }^{16,20}$ However, other studies found no significant difference in H-reflex measures after standing training. ${ }^{1,6}$ To the best of the authors' knowledge, this study was the first that compared spasticity changes after dynamic and static standing training in individuals with SCI. The findings of this study could not provide statistical evidence to support greater spasticity reduction after the dynamic standing training compared with the static standing training, but the data does show a remarkable reduction in spasticity immediately after dynamic standing compared with static standing for the gastrocnemius and hamstring muscles (Table 4).

The sample size was small for this study, thus the results should be interpreted with caution and the lack of significance is most likely due to the small sample size used in the study $(n=10)$. We acknowledge that a Type II error is possible. The graphical representation of the data does indicate that the dynamic training did produce a greater drop in spasticity as measured by the self report (VAS) and EMG. A larger sample size is clearly needed to know definitively whether dynamic training can be superior. We might be more optimistic to suggest it could be as useful as using a standing frame for the benefits toward spasticity. However, the additional benefits of using dynamic standing is when the individual helps with spasticity by standing and they can still get around going places and accomplishing mobility goals. ${ }^{10}$

Any study on spasticity is inherently challenging as spasticity is highly variable between days and is affected by the time of day, physical activity level, fatigue and medical conditions (for example, urinary tract infection). The timeline of the study limited the ability to control for the participants' physical activity while they participated in the study, and the small sample size limited the ability to control for participants with the same level of physical activity. However, this study did attempt to control for time of day between visits and requested participants refrain from changing their routines. It is possible that significant changes in daily routine physical activity level may have confounded the results. In addition, eight of the study participants had cervical injury (C3-C7), whereas only two had thoracic injury (T4-T6), such that the results are heavily influenced by effects in participants with cervical SCI. All but one of the study participants had Abbreviated Injury Scale score of $\mathrm{C}$ or D. The participant with Abbreviated Injury Scale score of A could finish the standing training with the help of standing bars on the side of the Segway but it may apply as a limitation to the study.

\section{CONCLUSION}

In conclusion, the dynamic standing training revealed no more beneficial effects for spasticity reduction versus the static standing training as measured by three different outcome measures: selfassessment (VAS), clinical (MAS), and electrophysiological (EMG). However, the similar effects of both dynamic and static training suggest that dynamic training may have some role in managing spasticity in individuals living with SCI.

\section{DATA ARCHIVING}

There were no data to deposit.

\section{CONFLICT OF INTEREST}

The authors declare no conflict of interest.

\section{ACKNOWLEDGEMENTS}

This research was funded by Natural Sciences and Engineering Research Council of Canada (NSERC) and International Collaboration on Repair Discoveries (ICORD). We acknowledge participation and help of the University of British Columbia undergraduate student Harleen Karla and graduate student Mehdi Eshraghi for data collection and completion of this research study.

1 Bakheit AMO, Maynard V, Shaw S. The effects of isotonic and isokinetic muscle stretch on the excitability of the spinal alpha motor neurones in patients with muscle spasticity. Eur J Neurol 2005; 12: 719-724.

2 Lance JW. The control of muscle tone, reflexes, and movement: Robert Wartenberg lecture. Neurology 1980; 30: 1303-1313.

3 Dietz V. Spasticity-spastic movement disorder. Spinal Cord 2008; 46: 588.

4 Young RR. Spasticity: a review. Neurology 1994; 44: S12-S20.

5 Decq P. [Pathophysiology of spasticity]. Neurochirurgie 2003; 49: 163-184.

6 Adams MM, Hicks AL. Spasticity after spinal cord injury. Spinal Cord 2005; 43: 577-586.

7 Newman M, Barker K. The effect of supported standing in adults with upper motor neurone disorders: a systematic review. Clin Rehabil 2012; 26: 1059-1077.

8 McGibbon CA, Sexton A, Jones M, O'Connell C. Elbow spasticity during passive stretch-reflex: clinical evaluation using a wearable sensor system. J Neuroeng Rehabil 2013; 10: 61.

9 Tsai $\mathrm{KH}$, Yeh $\mathrm{CY}$, Chang HY, Chen JJ. Effects of a single session of prolonged muscle stretch on spastic muscle of stroke patients. Proc Nat/ Sci Counc Repub China B 2001; 25: 76-81.

10 Sawatzky B, Denison I, Langrish S, Richardson S, Hiller K, Slobogean B. The segway personal transporter as an alternative mobility device for people with disabilities: a pilot study. Arch Phys Med Rehabil 2007; 88: 1423-1428.

11 Boutilier G, Sawatzky BJ, Grant C, Wiefelspuett S, Finlayson H. Spasticity changes in $\mathrm{SCl}$ following a dynamic standing program using the Segway. Spinal Cord 2012; 50: 595-598.

12 Adams MM, Hicks AL. Comparison of the effects of body-weight-supported treadmill training and tilt-table standing on spasticity in individuals with chronic spinal cord injury. J Spinal Cord Med 2011; 34: 488-494.

13 Hsieh JTC, Wolfe DL, Miller WC, Curt A, SCIRE Research Team. Spasticity outcome measures in spinal cord injury: psychometric properties and clinical utility. Spinal Cord 2008; 46: 86-95.

14 Sköld C, Harms-Ringdahl K, Hultling C, Levi R, Seiger A. Simultaneous Ashworth measurements and electromyographic recordings in tetraplegic patients. Arch Phys Med Rehabil 1998; 79: 959-965.

15 Segway Company Milestones [Internet]. (cited 30 Mar 2015). Available from http:// www.segway.com/about-segway/segway-milestones.php.

16 Field-Fote EC, Brown KM, Lindley SD. Influence of posture and stimulus parameters on post-activation depression of the soleus $\mathrm{H}$-reflex in individuals with chronic spinal cord injury. Neurosci Lett 2006; 410: 37-41.

17 Bohannon RW. Tilt table standing for reducing spasticity after spinal cord injury. Arch Phys Med Rehabil 1993; 74: 1121-1122.

18 Zupan B, Stokić DS, Bohanec M, Priebe MM, Sherwood AM. Relating clinical and neurophysiological assessment of spasticity by machine learning. Int J Med Inform 1998; 49: 243-251.

19 Laessøe L, Nielsen JB, Biering-Sørensen F, Sønksen J. Antispastic effect of penile vibration in men with spinal cord lesion. Arch Phys Med Rehabil 2004; 85: 919-924.

20 Kawashima N, Sekiguchi H, Miyoshi T, Nakazawa K, Akai M. Inhibition of the human soleus Hoffman reflex during standing without descending commands. Neurosci Lett 2003; 345: 41-44. 